\title{
Three-mode approximation of symmetrical triple-square wells
}

\author{
XinJian Liu, WeiDong $\mathrm{Li}^{*}$ \\ Institute of Theoretical Physics and Department of Physics, Shanxi University, Taiyuan, China \\ Email address: \\ 15364839097@163.com (XinJian Liu),wdli@sxu.edu.cn (WeiDong Li)
}

To cite this article:

XinJian Liu, WeiDong Li. Three-Mode Approximation of Symmetrical Triple-Square Wells. American Journal of Modern Physics. Vol. 3, No. 2, 2014, pp. 113-117. doi: 10.11648/j.ajmp.20140302.20

\begin{abstract}
One transformation, analogy to two mode approximation, is presented for triple-square wells. The energy splitting is determined by the strength of the tunneling coupling between nearest neighbor wells, while the next-nearest neighbor tunneling coupling plays crucial role to the invariant first excited state with the maximum entanglement states for the far separated square-wells.
\end{abstract}

Keywords: Symmetrical Triple-Square Wells, Three-Mode Approximation, Energy Spectrum, Transformation

\section{Introduction}

As a bridge between the simple double well and the multi-well systems, the triple-well system has been being extensively systems, the triple-well system has been being extensively investigated in various branches of physics, for example, adiabatic transport [1-4] in triple-well solid-state systems (quantum dots [1], ionized donors and superconductors [2]), and quantum phase transition and quantum dynamics in cold atoms with triple-well [5-9]. Compared with the double well, one more well provides a additional energy level to make solid systems realize one kind of electrons coherent population transfer, which amounts to transporting electron coherently from one end of well to the other by various methods, an analogy of Stimulated Raman Adiabatic Passage (STRIRAP) protocol in quantum optics (coherently transfer electron population between two long-lived atomic or molecular energy levels); in the other hand, the effect of next- nearest-neighbor coupling on quantum tunneling allows novel quantum dynamics, nonlinear Josephson oscillation [7], macroscopic quantum self trapping and dipolar quantum gases has been explored in cold atom field [10]. In coherent tunneling adiabatic passage of solid-state system, macroscopic quantum self trapping and even quantum phase transition in cold atom physics, spatial dark state plays an important role and can be determined from the energy spectrum of triple-well. Similar as in double well [11-13], the three modes approximation is expected to provide a picture to understand the nature of these processes. For double well case, the original degenerate ground states for each single well are split into the two lowest global energy levels by tunneling mechanism. The strength of the coupling determines the energy gap (called as energy splitting in some sense) between the lowest two global energy levels. Two mode approximation provides a transformation by which the localised two modes (approximated to the ground states of single well) can be obtained from the two lowest global levels. It is natural to look for one similar transformation for triple-well case, by which the localised three modes, both wave-functions and eigenvalues can be obtained from the three lowest global levels.

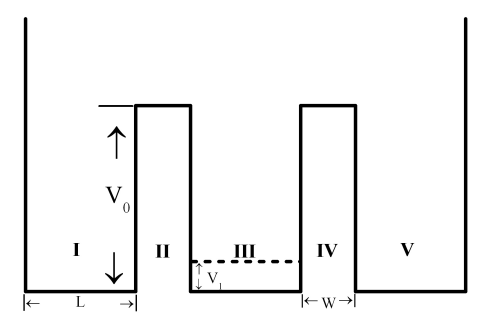

Figure 1. Triple-square well and adjusting biased energy $V_{1}$.

In this manuscript, we are going to develop this kind of transformation by considering a triple square wells. With the help of the analytical solution, the validity of this transformation is checked, both for the eigenvalues and wave-functions. A two tunneling procedures, each one consisting with two levels tunneling coupling, are suggested to understand the forming process for three lowest global levels. The energy splitting between the 
ground state and first excited state, and the first and the second excited state is determined by strength of tunneling coupling between nearest neighbour wells, but next-nearest neighbour tunneling coupling (very weak than the nearest neighbour one) plays an crucial role to realize the first excited state, being the maximum entanglement states.

Our manuscript is organised as following. In Sec. II, we introduce the mode and analytical solution, the Energy spectrum varied with the width of the barrier. One transformation is presented Sec.III, and its validity is checked by calculating the eigenvalue and eigen-functions, and two tunneling procedures are suggested to understand the invariant first excited state.

\section{Mode and Energy Spectrum}

The one-dimensional triple-square wells can be composed by the two semi-infinite square and one finite square well (see Fig. 1). The energy spectrum and corresponding the following one dimensional Schrodinger equation

$$
\left[-\frac{\partial^{2}}{\partial x^{2}}+V(x)\right] \Phi(x)=E \Phi(x)
$$

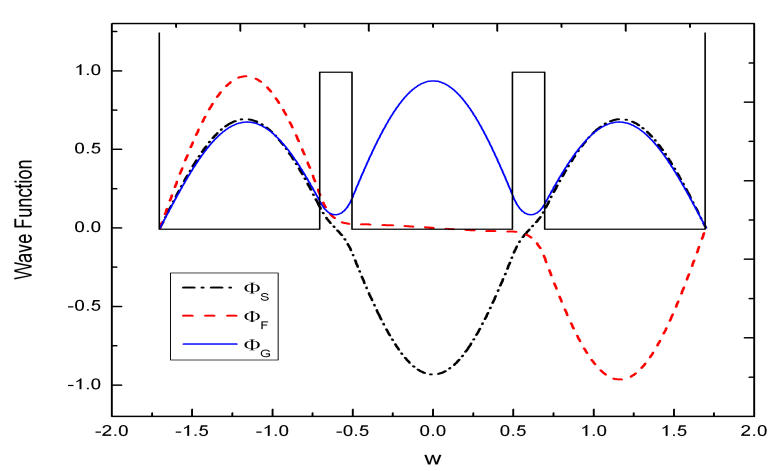

Figure 2. One example of eigen-functions for the lowest three global energy levels for triple-square well.

where $E$ is the eigenvalue for given eigen wave function $\Phi(x)$. The external potential $V(x)$ can be expressed as

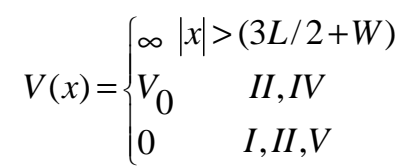

where $V_{0}$ and $w$ denote the Barrier height and width respectively; $L$ is the width of square well, which play as the typical length in our paper, and the related energy unit is defined by $\hbar^{2} / 2 m L^{2}$.

It is natural to solve stationary Schrodinger equation

(1) by assuming $\Phi(x)$ as in region $I, I I I$ and $V$

$$
f_{i}(x)=A_{i} \sin \left(k\left(x+\gamma_{i}\right)\right)
$$

and in regions of $I I, I V$,

$$
g_{j}(x)=B_{j 1} e^{-\beta x}+B_{j 2} e^{\beta x}
$$

where $i=1,2,3$ for regions $I, I I I$ and $V$, and $j=1,2$ for regions $I I$ and $I V, k=\sqrt{E}$ and $\beta=\sqrt{V_{0}-E}$.

Considering the boundary conditions at $x= \pm 3 L / 2 \pm w$, we have, $\gamma_{1}=3 L / 2+w$ and $\gamma_{3}=-3 L / 2-w$. Other parameters $A_{1}, B_{11}, B_{12}, A_{2}, B_{21}, B_{22}, A_{3}, E, \gamma_{2}$ can be fixed by solving the matching conditions at $x_{0}^{\prime}= \pm(L / 2+w), x_{1}^{\prime}= \pm L / 2$, and normalization condition

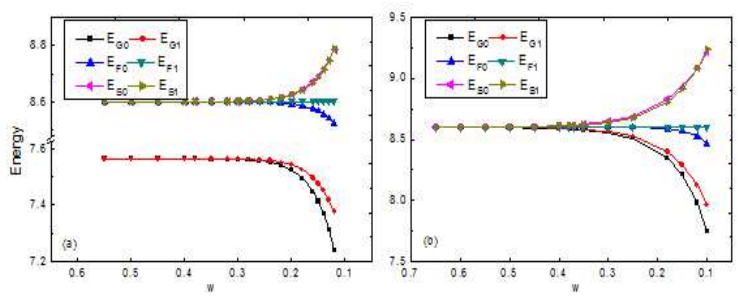

Figure 3. The energy spectrum (for lowest three energy levels) with width of barrier for $E_{1} \neq E_{0}(a)$, and $E_{1}=E_{0}(b)$.

$$
\begin{aligned}
& f_{i}\left(x^{\prime}\right)=g_{i}\left(x^{\prime}\right), f_{i}^{\prime}\left(x^{\prime}\right)=g_{j}^{\prime}\left(x^{\prime}\right), \\
& \sum_{i=1,2,3} \int_{i}\left|f_{i}(x)\right|^{2} d x+\sum_{j=1,2} \int_{j}\left|g_{j}(x)\right|^{2} d x=1 .
\end{aligned}
$$

First of all, we can find the ground state of each single square wells (two semi-infinite $\left(\phi_{1}(x)\right.$ with $\left.E_{1}\right)$ and one finite square well $\left(\phi_{0}(x)\right.$ with $\left.\left.E_{0}\right)\right)$, by using similar assumption. It is interesting to mention that $E_{1} \neq E_{0}$, for same $V_{0}$. This makes us classify the triple-square wells as two categories. Both of them are geometrical symmetry as shown in Fig. 1, but one with the different ground energies $\left(E_{1} \neq E_{0}\right)$, other one with same ground energies $\left(E_{1}=E_{0}\right)$ by adjusting one additional potential $V_{1}$ (see Fig. 1).

Then arranging the three single square wells as Fig.1 and adjusting width $w$ of the barrier, we can find the global lowest three eigen energies by solving the matching condition (3). In Fig. 3, the relations of the corresponding energy spectrum with $w$ have been plotted by setting $V_{0}=200, L=1$, wherein Fig.(3) (a) is for $E_{1} \neq E_{0}$ and Fig.(3) (b) is for $E_{1}=E_{0}$. With decreasing $w$, the coupling between neighbor square wells is enhanced by tunneling mechanism, and a energy splitting is found once $w$ is smaller than some critical values $w_{c}$. As one example, we also show the eigen-functions, in Fig. 2 for $w=0.2$, where the solid curve is for the ground state energy $\left(E_{G}\right)$ with wave function $\left(\Phi_{G}\right)$, the dashed one for the first excited state energy $\left(E_{F}\right)$ with wave function $\left(\Phi_{F}\right)$ and the dotted line for the second excited state energy $\left(E_{S}\right)$ with wave function $\left(\Phi_{S}\right)$. 


\section{Two Tunneling Procedures and Invariant First Excited State}

Analogies with double wells cases, we are going to look for one transformation by which the global eigen-states ( $\Phi$, shown in Fig.3) can be constructed by the three, localized ground states $\left(\phi_{i}\right)$ of each single square wells, therefore, called as three-modes approximation. Following the same procedures as in the two-modes approximation. Following the same procedures as in the two-mode (symmetrical double well) case, a transformation matrix $S$ can be written as

$$
S=\left(\begin{array}{ccc}
\frac{e}{\sqrt{2 e^{2}+\Delta_{G}^{2}}} & -\frac{\Delta_{G}}{\sqrt{2 e^{2}+\Delta_{G}^{2}}} & \frac{e}{\sqrt{2 e^{2}+\Delta_{G}^{2}}} \\
\frac{1}{\sqrt{2}} & 0 & -\frac{1}{\sqrt{2}} \\
\frac{e}{\sqrt{2 e^{2}+\Delta_{S}^{2}}} & \frac{\Delta_{S}}{\sqrt{2 e^{2}+\Delta_{S}^{2}}} & \frac{e}{\sqrt{2 e^{2}+\Delta_{S}^{2}}}
\end{array}\right)
$$

By which the global eigen functions $\Phi=\left(\Phi_{G}, \Phi_{F}, \Phi_{S}\right)$ can be constructed as

$$
\Phi=S \cdot \phi
$$

Where $\phi=\left(\phi_{1}, \phi_{2}, \phi_{3}\right)^{T}$ is the local or ground states of single square wells. The corresponding Hamiltonian $H$ are expressed as

$$
H=\left(\begin{array}{ccc}
E_{1} & e & e_{1} \\
e & E_{0} & e \\
e_{1} & e & E_{1}
\end{array}\right)
$$

Where $E_{1}$ and $E_{0}$ are defined as before, and are the ground states of the side of triple-square well(semi-infinite square well) and the middle one (finite deep potential well) respectively, and $e$ and $e_{1}$ denote the tunneling coupling between the neighbor wells and defined as $e=-\left\langle\phi_{1}(x)\left|-\frac{\partial^{2}}{\partial x^{2}}+V(x)\right| \phi_{2}(x)\right\rangle, e_{1}=-\left\langle\phi_{1}(x)\left|-\frac{\partial^{2}}{\partial x^{2}}+V(x)\right| \phi_{3}(x)\right\rangle \cdot$

It is easy to prove that $e_{1}$ is usually much smaller than $e$ and can be neglected, since those two wells are far separated by middle one. Therefore, the lowest three global eigen states can be expressed as

$$
\begin{aligned}
& \Phi_{G}=\frac{e}{\sqrt{2 e^{2}+\Delta_{G}^{2}}}\left(\phi_{1}(x)-\frac{\Delta_{G}}{e} \phi_{2}(x)+\phi_{3}(x)\right), \\
& \Phi_{F}=\frac{1}{\sqrt{2}}\left(\phi_{1}(x)-\phi_{3}(x)\right), \\
& \Phi_{S}=\frac{e}{\sqrt{2 e^{2}+\Delta_{S}^{2}}}\left(\phi_{1}(x)+\frac{\Delta_{S}}{e} \phi_{2}(x)+\phi_{3}(x)\right),
\end{aligned}
$$
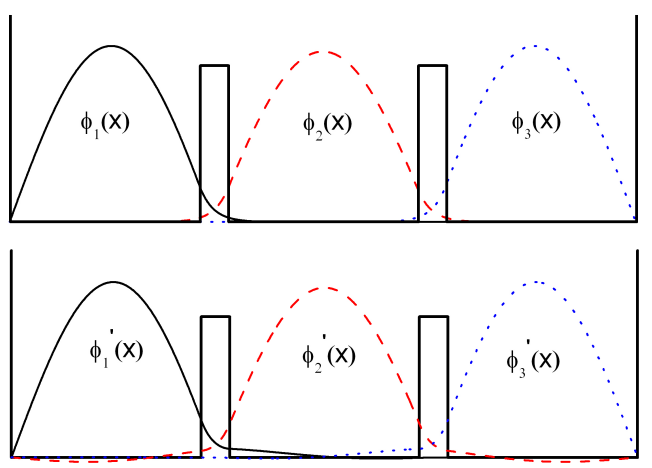

Figure 4. Localised wave function (bottom) obtained from Eq. (10) and ground states for each single square wells (top).

during the process $e_{1}$ has been neglected, $\Delta_{G}=E_{G}-E_{F}$ and $\Delta_{S}=E_{F}-E_{S}$. It is interesting to note that the similar results as (7) has been obtained in [1].

Table 1. The eigen-values of lowest three states.

\begin{tabular}{lllll}
\hline $\mathbf{w}$ & $\mathbf{0 . 5}$ & $\mathbf{0 . 4}$ & $\mathbf{0 . 1 5}$ & $\mathbf{0 . 1 2}$ \\
\hline$E_{G 0}$ & 7.56341 & 7.56326 & 7.41303 & 7.24193 \\
$E_{G 1}$ & 7.56341 & 7.56334 & 7.47646 & 7.37639 \\
Percentage & 0 & 0.00106 & 0.84840 & 1.82280 \\
$E_{F 0}$ & 8.60075 & 8.60085 & 8.69148 & 8.52232 \\
$E_{F 1}$ & 8.60075 & 8.60075 & 8.60075 & 8.60075 \\
percentage & 0 & 0.00035 & 0.39264 & 0.91190 \\
$E_{S 0}$ & 8.60075 & 8.60085 & 8.69148 & 8.78778 \\
$E_{S 1}$ & 8.60075 & 8.60083 & 8.68771 & 8.78381 \\
percentage & 0 & 0.00023 & 0.04339 & 0.04518 \\
\hline
\end{tabular}

The valid of relations (4) can be easily checked with the help of the global eigen states for triple-square well and the single square well. In Table 1, we list the lowest three global eigen energies with a few width $w$, where $E_{\ldots 0}$ denotes the values by solving the matching condition (3) and $E_{\ldots 1}$ is found by

$$
\begin{aligned}
& E_{G}=\frac{\left(E_{1}+E_{0}\right)-\sqrt{\left(E_{1}-E_{0}\right)^{2}+8 e^{2}}}{2}, \\
& E_{F}=E_{1}, \\
& E_{S}=\frac{\left(E_{1}+E_{0}\right)+\sqrt{\left(E_{1}-E_{0}\right)^{2}+8 e^{2}}}{2} .
\end{aligned}
$$

Which is obtained by (5) based on the knowledge of ground states of the single square wells $\phi_{i}(x)$.Around the region $w \sim 0.2$, the ratio of the tunneling $e$ can be considered as a small quantity, compared with $E_{1}-E_{0}$,we can have a simple relations from (8), 


$$
\begin{aligned}
& E_{G} \sim E_{0}-\frac{2 e^{2}}{\left(E_{1}-E_{0}\right)}, \\
& E_{F}=E_{1}, \\
& E_{S} \sim E_{1}+\frac{2 e^{2}}{\left(E_{1}-E_{0}\right)} .
\end{aligned}
$$

Further decreasing the width of barrier, one clear deviation can be found in the region $w>0.25$ in Fig.3. This may reveal the valid region for those three modes approximation or our simplified modes $(\phi(x))$ are not good enough.

But we still can have the localized modes from the inverse transformation of (5), by which we have the localized modes from the global lowest three levels with

$$
\phi^{\prime}(x)=S^{-1} \Phi(x)
$$

where all elements in $S$ are defined by the information of global modes, means $\Delta_{G}=E_{G 0}-E_{F 0}, \Delta_{S}=E_{F 0}-E_{S 0}$ and $e=\sqrt{\Delta_{G} \Delta_{S} / 2}$. Similarly, we check the valid of (10) by calculating the following fidelity

$$
F_{i}=\int \phi_{i}(x) \phi_{i}^{\prime}(x) d x
$$

As one example, the localized modes $\phi_{i}^{\prime}(x)$, based on (10) are plotted for $w=0.15$ in Fig. 4. Once the barrier is narrow, for example $w=0.15 \quad$ we have $F_{1,3}=0.99431, F_{2}=0.99657$, which shows the deviation of the energy spectrum too. While in the wide barrier, for example $w=0.38$, a good consistent of the localized functions with the ground states can be found from the high fidelity $F_{1,3}=0.999999, F_{2}=0.999999$.

It is interesting to note that

$$
e=\sqrt{\frac{\Delta_{G} \Delta_{S}}{2}}
$$

from the requirement of the orthogonal conditions of (4). Similar with the two-mode approximation, the energy splitting between the ground states and excited states are defined by the strength of the tunneling coupling. When $w$ is large, each square well can be considered as one complete separated wells. In this case, $e$ is small enough and can be safely neglected, we have two degenerate two square wells $\left(E_{F}=E_{S}=E_{1}\right.$ ), but separated by one finite square well with lower energy $E_{G}=E_{0}<E_{1}$. This is exactly what we shown in Fig.3 for $w>0.25$. With decreasing the value of $w$, the tunneling coupling is increasing, and the energy splitting $\Delta_{G, S}$ are found.

Further investigation, we found the following two tunneling procedures

$$
S=S_{2} \cdot S_{1}
$$

Where

$$
S_{1}=\frac{\sqrt{2}}{2}\left(\begin{array}{ccc}
1 & 0 & 1 \\
0 & \sqrt{2} & 0 \\
1 & 0 & -1
\end{array}\right) \text {, }
$$

and

$$
S_{2}=\frac{\sqrt{2}}{2}\left(\begin{array}{ccc}
\frac{\sqrt{2 e}}{\sqrt{2 e^{2}+\Delta_{G}^{2}}} & \frac{-\Delta_{G}}{\sqrt{2 e^{2}+\Delta_{G}^{2}}} & 0 \\
\frac{0}{\sqrt{2 e}} & \frac{\Delta_{S}}{\sqrt{2 e^{2}+\Delta_{S}^{2}}} & 0
\end{array}\right) \text {. }
$$

Eq. (13) reveals a two tunneling procedures to form the final global eigen states from the single square wells: Firstly, the two far separated semi-square wells are coupled through $S_{1}$, during which the middle well keeps its original properties; The second process is a two levels coupling too, where the lowest energy level, obtained in the first process, is coupled with the ground state of middle square well. Both process are tunneling coupling, similar with the two modes approximation. It is interesting to mention that even the coupling between the first process is always smaller than the second one (due to far separated), but it prepares the coupling energy level for the second process.

After analysing the geometrical symmetry triple square wells, one simple relation (5) is obtained to connect the global and localised modes. This relation can be easily to extend to the total symmetry case, means the energy also exactly same for those three single square wells. This could be easily to realised by experimentally adjusting the bias voltage $V_{1}$ applied on the middle quantum dot [2]. Theoretically, this can be obtained by asking

$$
\sqrt{E_{1}-V_{1}} * \tan \left(\frac{L \sqrt{E_{1}-V_{1}}}{2}\right)=\sqrt{V_{0}-E_{1}},
$$

Where $V_{1}$ is additional constant potential in region III. With same procedures, we have the energy spectrum for those symmetry triple-square wells, shown in Fig. 3 (b), where $V_{1}=1.04232$ are used, other situation are the same as in the previous one.

Table 2. The eigen-values of ground states.

\begin{tabular}{llllll}
\hline $\mathbf{W}$ & $\mathbf{0 . 6 5}$ & $\mathbf{0 . 5}$ & $\mathbf{0 . 4}$ & $\mathbf{0 . 1 5}$ & $\mathbf{0 . 1 2}$ \\
\hline$E_{G 0}$ & 8.60039 & 8.59792 & 8.55528 & 8.20980 & 7.74365 \\
$E_{G 1}$ & 8.60049 & 8.59859 & 8.56351 & 8.28838 & 7.96297 \\
percentage & 0.00122 & 0.00784 & 0.09611 & 0.94807 & 2.75418 \\
\hline
\end{tabular}

Due to this energy symmetry, we found $\Delta_{G}=\Delta_{S}=-\sqrt{2} e$, and substitute it into (4) we have 


$$
S=\frac{1}{2}\left(\begin{array}{ccc}
1 & \sqrt{2} & 1 \\
\sqrt{2} & 0 & -\sqrt{2} \\
1 & -\sqrt{2} & 1
\end{array}\right),
$$

and similar two steps transformation matrix can be expressed as $S^{\prime}=S_{2}^{\prime} \cdot S_{1}$, where

$$
S_{2}^{\prime}=\frac{\sqrt{2}}{2}\left(\begin{array}{ccc}
1 & 1 & 0 \\
0 & 0 & \sqrt{2} \\
1 & -1 & 0
\end{array}\right) \text {. }
$$

Finally, we emphasis that the first excited state $\Phi_{F}$ keeps its the value ( $E_{1}$, eigen values of semi-infinite square well) and does not change anymore with decreasing $w$ for both cases. The global first excited state in this triple-square wells case can be expressed as

$$
\Phi_{F}=\frac{\sqrt{2}}{2}\left(\phi_{1}(x)-\phi_{3}(x)\right),
$$

where, the $\phi_{1,3}(x)$ is nothing but the ground state of the left (right) potential well. This can be easily understood from the two steps process, wherein the first excited states after the first coupling process by $S_{1}$ is survival and keeps away in the following second coupling process. In the point of view of quantum, this excited state is the maxima entangle state between those two loclized wave functions [14-16]. Therefore, this may suggest one way to create a maxima entanglement state between those far separated square wells. In other words, once our system is prepared in its first excited state, then we actually simultaneous got one maximum entanglement state.

\section{Conclusion}

We have investigated the three lowest states energy spectrum and its corresponding eigen-functions for triple-square well. With the analytical solutions for triple-square wells, we found a tree-mode approximation, by which the three local wave functions (modes) can be constructed from the global lowest states (similar to the two square wells case). Furthermore, a two tunneling process has been suggested to understand the forming process of the three lowest energy spectrum. One simple energy splitting relations between the ground state and first excited state are obtained. With this two tunneling procedures, we explain how its first excited state is formed and demonstrate that it is the maximum entangled states for the far separated square-wells.

This work was supported by the NNSFC (No.11374197, 11074155, 10934004), PCSIRT (No.IRT13076), National Programs for High Technology Research and Development of China (Grant No.2011AA010801).

\section{References}

[1] Andrew D. Greentree, Jared H. Cole, A. R. Hamilton, and Lloyd C. L. Hollenberg, Coherent electronic transfer in quantum dot systems using adiabatic passage, Phys. Rev. B. 70,235317 (2004)

[2] J. H. Cole, A. D. Greentree, L. C. L. Hollenbeg, and S. Das. Sarma, Spatial adiabatic passage in a realistic triple well structure, Pyhs. Rev. B. 77, 235418 (2008)

[3] Xin-You $\mathrm{Lu}$ and Jing $\mathrm{Wu}$, Three-mode entanglement viatunneling-induced interference in a coupled triple-semiconductor quantum-well structure, Pyhs. Rev. A. $82,012323(2010)$

[4] Jero Me Rech and Stefan Kehrein, Effect of Measurement Backaction on Adiabatic Coherent Electron Transport, Phys. Rev. Lett. 106,136808 (2011)

[5] B. Liu, L. B. Fu, S. P. Yang, and, J. Liu, Josephson oscillation and transition to self-trapping for Bose-Einstein condensates in a triple-well trap, Phys. Rev. A.75, 033601 (2007)

[6] WangHai-Lei, YangShi-Ping, Switch effect of Bose-Einstein condensates in a triple-well potential, Acta. Phys. Sin. 57, 3290(2008)

[7] Rosario Paredes, Tunneling of ultracold Bose gases in multiple wells, Phys. Rev. A. 73, 033616 (2006)

[8] Thiago F Viscondi, and, K Furuya, Dynamics of Bose-Einstein condensate in a symmetric triple-well trap,J. Phys.A.44,1753 (2011)

[9] Alexej I. Streltsov, Kaspar Sakmann, Ofire. Alon and Lorenz. S. Cederbaum, Accurate multi-boson long-time dynamics in triple-well periodic traps, Phys. Rev. A. 83, 043604 (2011)

[10] T. Lahaye, T. Pfau, and, L. Santos, Mesoscopic Ensembles of Polar Bosons in Triple-Well Potentials, Phys. Rev. Lett. 104,170404 (2010)

[11] A. Smerzi, S. Fantoni, S. Giovanazzi, and,S. R. Shenoy, Quantum Coherent Atomic Tunneling between Two Trapped Bose-Einstein Condensates,Phys. Rev. Lett. 79,4950 (1997)

[12] WeiDong Li, Stationary solutions of Gross-Pitaevskii equations in a double square well, Phys. Rev. A. 74, 063612 (2006)

[13] XinYan Jia, WeiDong Li, and, J. Q. Liang, Nonlinear correction to the boson Josephson-junction model, Phys. Rev.A. 78, 023613 (2008)

[14] Ping-Zhang, Xian-Geng Zhao, Localization and entanglement of two interacting electrons in a double quantum dot, J. Phys. Condens. Matter.13, 8389 (2001)

[15] S. J. Van. Enk, Pyhs. Single-particle entanglement, Rev. A. 72, $064306(2005)$

[16] Marcelo O. Terra Cunha, Jacob A Dunningham and Vlatko Vedral, Proc. R. Soc. A. 463, 2277 (2007) 